\title{
Caracterización de Estructuras Anatómicas Mediante Radiografías Panorámicas: El Foramen Mental
}

\author{
Characterization of Anatomical Structures Using Panoramic Radiographs: The Mental Foramen
}

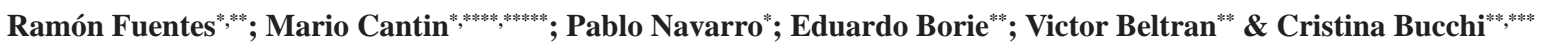

FUENTES, R.; CANTIN. M.; NAVARRO, P.; BORIE, E.; BELTRAN, V. \& BUCCHI, C. Caracterización de estructuras anatómicas mediante radiografías panorámicas: El foramen mental. Int. J. Morphol., 32(4):1423-1429, 2014.

RESUMEN: El foramen mental (FM) es un referente anatómico importante durante procedimientos quirúrgicos y colocación de implantes. Con el propósito de conocer la detectabilidad y posición del FM, caracterizándolo respecto a sus relaciones anatómicas, fueron analizadas 215 radiografías panorámicas convencionales. Además, se determinó la presencia y extensión del bucle del canal mandibular. El FM se detectó en el 87,9\% de los casos, en mayor porcentaje en hombres y a menor edad. La posición anteroposterior más frecuente del FM fue en relación al eje longitudinal del segundo premolar en el lado derecho y en el izquierdo entre los ejes longitudinales del primer y segundo premolar. La posición supero-inferior más frecuentemente observada en ambos sexos y todos los grupos etarios fue bajo el ápice de los dientes. La distancia promedio desde el centro del FM a la línea mediana fue de 35,3 mm y 35,6 mm en el lado derecho e izquierdo, respectivamente. La distancia promedio desde el centro del FM al margen mandibular fue de 16,6 mm a la derecha y 17,1 mm a la izquierda. En relación al bucle del canal mandibular, se detectó en un 33,9\% de los casos y su extensión fue de 5,3 mm y $4,8 \mathrm{~mm}$ en el lado derecho e izquierdo, respectivamente. Es fundamental conocer la anatomía, ubicación de estructuras y evaluar esta zona previo a cualquier procedimiento quirúrgico o colocación de implantes dentales, para así evitar lesiones del FM y nervio mental o el bucle del canal mandibular anterior o posterior y nervio alveolar inferior.

PALABRAS CLAVE: Foramen mental; Canal mandibular; Bucle anterior del canal mandibular; Nervio alveolar inferior; Radiografía panorámica.

\section{INTRODUCCIÓN}

El canal mental e incisivo provienen de la bifurcación del canal mandibular, el cual contiene ramos y ramas de los nervio, arterias y venas alveolares inferiores (Olivier, 1928). Anatómicamente, el foramen mental (FM) se encuentra en la pared bucal del cuerpo de la mandíbula, marcando el término del canal mental por el cual emerge el paquete neurovascular que da inervación e irrigación a los tejidos blandos de la zona bucal, labio inferior y mentón (Standring et al., 2005). Es fundamental conocer la anatomía de esta zona, principalmente por los procedimientos quirúrgicos que la involucran, tales como toma o colocación de injertos óseos, apicectomías o implantes dentales, y evitar así lesionar estructuras que la comprometen.

La literatura describe frecuentemente la ubicación del FM mediante el uso de radiografías panorámicas, las cuales tienen una capacidad de detectar esta estructura por sobre el 95\% (al Jasser \& Nwoku, 1998; Ngeow \& Yuzawati, 2003). Produce una imagen de calidad diagnóstica suficiente para permitir una visión inmediata de todo el sistema maxilofacial del paciente, a pesar de una cierta distorsión y magnificación. Por esta razón, la radiografía panorámica se considera que es el estándar de examen radiográfico para la planificación del tratamiento de implante. Además, posee ventajas respecto a la radiografía periapical, como mayor área de visualización y capacidad de posicionar al FM respecto a estructuras vecinas. Otra alternativa son las tomografías computarizadas, que si bien ofrecen una excelente visualización de la mandíbula en tres dimensiones, tiene deficiencias como la radiación y el costo económico superior; y su uso no está tan difundido en la práctica odontológica (Aminoshariae et al., 2014).

* Programa de Magister en Odontología, Facultad de Odontología Universidad de La Frontera, Temuco, Chile.

** Centro de investigación CIMOFIR, Facultad de Odontología, Universidad de La Frontera, Temuco, Chile.

*** Alumna Programa de Magister en Odontología, Facultad de Odontología, Universidad de La Frontera, Temuco, Chile.

***** Programa de Doctorado en Ciencias Morfológica, Facultad de Medicina, Universidad de La Frontera, Temuco, Chile.

****** Centro de Investigación en Ciencias Biomédicas, Universidad Autónoma de Chile, Temuco, Chile. 
Se han reportado diferencias en la posición del FM en relación al tipo de población estudiada (Igbigbi \& Lebona, 2005; Al-Khateeb et al., 2007; Yesilyurt et al., 2008; al Jasser \& Nwoku; Ngeow \& Yuzawati; Singh \& Srivastav, 2010), edad (Al-Khateeb et al.), sexo (Al-Khateeb et al.; Amorim et al., 2008) y estado de la dentición (Amorim et al.), entre otros factores. La mayoría de estos estudios concuerda que la posición más frecuente se encuentra en relación a la línea con el eje longitudinal del segundo premolar o entre el primer y segundo premolar. También existen diferencias entre los autores con respecto a la presencia (Yosue \& Brooks 1989; Jacobs et al., 2004) y extensión del bucle o loop del canal mandibular. Se han reportado longitudes de entre $0,8 \mathrm{~mm}$ (Uchida et al., 2009) y $11 \mathrm{~mm}$ (Neiva et al., 2004) en cráneos secos.

El objetivo de este estudio fue determinar la detectabilidad del foramen mental y bucle del canal mandibular y caracterizarlo respecto a sus relaciones anatómicas mediante radiografías panorámicas en una muestra de pacientes chilenos adultos.

\section{MATERIAL Y MÉTODO}

Se realizó un estudio de tipo descriptivo y corte transversal. Fueron analizadas 215 radiografías panorámicas convencionales provenientes de una clínica particular de la ciudad de Temuco, Chile, entre los años 2003 y 2012. Las radiografías fueron tomadas con la técnica estándar con un Ortopantomógrafo (Proline CC, Planmeca, Helsinki, Finlandia) y su proceso de revelado se efectuó en una reveladora automática (Durr, XR 24 pro, Bietigheim-Bissingen, Alemania) (Placas radiográficas Kodak, T- MAT G/RA, Nueva York, EEUU). Todas las radiografías analizadas (magnificación 30\%) en el estudio pertenecían a pacientes con edad y sexo conocido. Se excluyeron radiografía de pacientes menores de 21 años, con distorsión o alteración en el contraste, presencia de patología, dientes incluidos, enanismo radicular o presencia de placas de titanio en el área de ubicación del FM. La muestra se conformó de 215 radiografías (215 lados derechos y 214 lados izquierdos) de pacientes con edades entre 21 y 82 años. La distribución según sexo fue 73 hombres y 142 mujeres. Las radiografías fueron clasificadas según sexo, edad y lado.

Se determinó la presencia y posición del FM. Para estudiar su posición supero-inferior y antero-posterior respecto a los dientes, se analizaron sólo los datos de FM detectables y que presentaran continuidad dentaria mandibular en la región premolar, tanto en posición mesial como distal en relación al FM (126 en el lado derecho y 119 izquierdo). La clasificación posicional del FM utilizada en el estudio se observa en la Tabla I y Figura 1. La posición del FM fue establecida de acuerdo al hueso mandibular, para lo cual se analizaron exclusivamente los lados con el FM visible (186 y 191 hallazgos, en el lado derecho e izquierdo, respectivamente), utilizando para realizar las mediciones un negatoscopio y se llevó a cabo un trazado manual en una placa de acetato puesta sobre la placa radiográfica (Fig. 2), diseñando las siguientes líneas:

- Una línea tangente al margen inferior del cuerpo de la mandíbula, paralela a la horizontal (L1) (Fuentes et al., 2008).

- Una línea a través de la línea mediana determinada por la espina nasal anterior y perpendicular a L1 (L2) (Fuentes et al.).

- Una línea desde el centro del foramen mental hasta la línea L1 (L3).

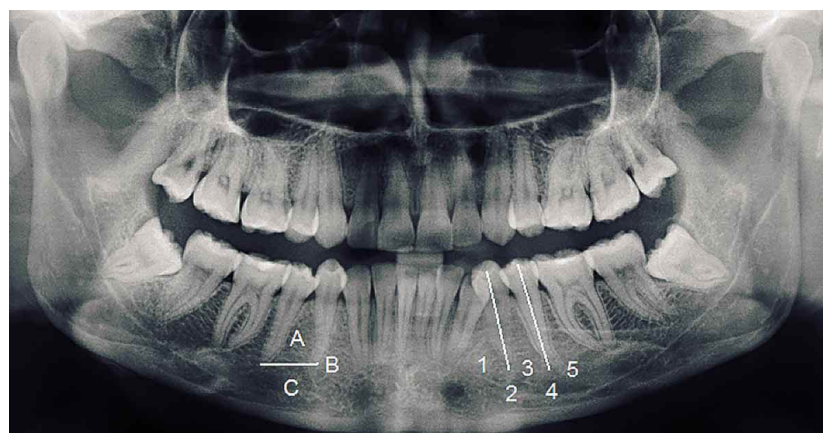

Fig. 1. Radiografía panorámica con la posición supero-inferior del foramen mental $(\mathrm{A}-\mathrm{C})$ y la posición antero-posterior (1-5) con relación a los dientes; descripción de la información contenida en la Tabla I.

Tabla I. Ubicación supero-inferior y antero-posterior del foramen mental en relación a los dientes.

\begin{tabular}{ll}
\hline Posición antero- & (1) Anterior al eje longitudinal del primer premolar. \\
posterior de foramen & (2) En línea con el eje longitudinal del primer premolar. \\
en relación a los & (3) Entre los ejes longitudinales del primer y segundo premolar. \\
dientes & $\begin{array}{l}\text { (4) En línea con el eje longitudinal del segundo premolar. } \\
\text { (5) Posterior al eje longitudinal del segundo premolar }\end{array}$ \\
$\begin{array}{l}\text { Posición supero- } \\
\text { inferior del foramen }\end{array}$ (A) Coronal al ápice o coronal a la línea que una los ápices de los dientes en donde se encuentre el \\
en relación a los \\
dientes
\end{tabular}


A partir de estos trazados se determinó la distancia desde el centro foramen a la línea mediana (D1) y al margen inferior de la mandíbula (D2) (Fig. 2).

Además, se estudió la presencia y extensión del bucle del canal mandibular; a los trazados anteriores se agregó:

- Una línea (L4) tangente al punto más anterior del bucle del canal mandibular y paralela a L3 (Fig. 2). La longitud del bucle se definió como D3 (distancia entre L3 y L4) (Fig. 2).

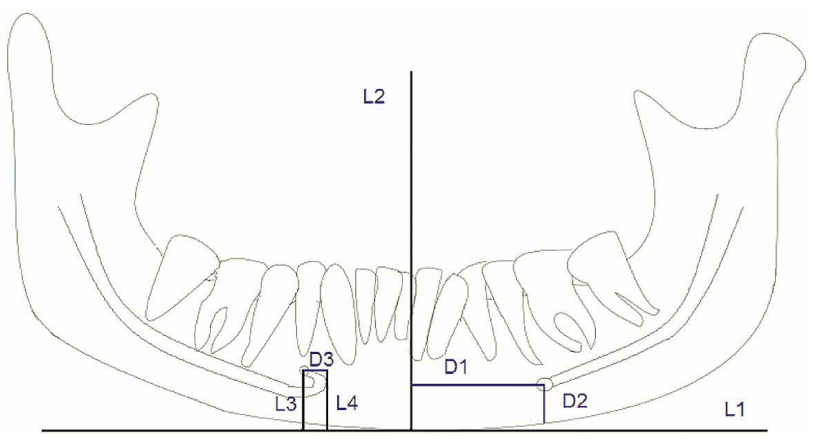

Fig. 2. Esquema de mandíbula con las líneas de medición, mostrando el trazado de la distancia del foramen al margen inferior de la mandíbula (D1), a la línea mediana (D2) y la extensión del bucle del canal mandibular (D3).

Las mediciones se realizaron con un Cáliper digital marca Mitutoyo (Mitutoyo America Corporation, Illinois, EEUU) con una precisión $\pm 0,02 \mathrm{~mm}$, por un único operador que realizó las mediciones en dos ocasiones, existiendo 7 días entre la primera y segunda medición. En los casos en que no existió concordancia entre la primera y segunda medición, se realizó una tercera medición, la cual se estableció como definitiva.

El análisis estadístico se realizó utilizando el software (SPSS/PC + versión 20.0, Chicago, IL). La estadística descriptiva fue calculada para las diferentes mediciones, entre ellas la media y desviación estándar. La homogeneidad de varianza se determinó a través de la Prueba de Levene y se utilizó la prueba Chi-Cuadrado de Pearson y el ANOVA de un factor. En todos los análisis estadísticos fue utilizado un nivel de significancia de $\mathrm{p} \leq 0,05$.

\section{RESULTADOS}

El FM fue detectado en un 87,9\% de los casos. Entre los gupos etáreos analizados, se observó que a mayor edad existe una menor detección del FM, diferencia estadísticamente significativa tanto al lado derecho de la mandíbula $(\mathrm{p}=0,001)$ como en el izquierdo $(\mathrm{p}=0,01)$. Se encontró un menor hallazgo del FM en pacientes de sexo femenino, observando solamente al lado izquierdo diferencias estadísticamente significativas $(\mathrm{p}=0,02)$. Esta información se encuentra detallada en la Tabla II.

La posición anteroposterior más frecuente del foramen mental en el lado derecho de la mandíbula, fue en relación a la línea con el eje longitudinal del segundo premolar $(50,8 \%)$ (Posición 4, Tabla I) y en el lado izquierdo fue entre los ejes longitudinales del primer y segundo premolar (44,5\%) (Posición 3, Tabla I). La posición superoinferior del foramen más frecuentemente observada en ambos sexos y en todos los grupos etarios fue bajo el ápice de los dientes (Posición C, Tabla I). El detalle de los análisis son descritos en detalles en las Tablas III y IV.

La distancia promedio (Media \pm DE) desde el centro del FM a la línea mediana (D1) fue de $35,3 \pm 5,1 \mathrm{~mm}$ y $35,6 \pm 5,4 \mathrm{~mm}$ en los lados derecho e izquierdo, respectivamente. La distancia promedio desde el centro del FM al margen mandibular (D2) fue de $16,6 \pm 2,5 \mathrm{~mm}$ en el lado derecho y $17,1 \pm 2,3 \mathrm{~mm}$ en el lado izquierdo.

Con relación al bucle del canal mandibular, éste fue detectado en posición anterior en un 33,9\% de los lados, siendo identificado en un $27 \%$ en el lado derecho $(n=51)$ y en un $41 \%$ en el lado izquierdo $(\mathrm{n}=75)(\mathrm{p}=0,00)$. La extensión del bucle (D3) (Media \pm DE) fue de 5,3 $\pm 2,2 \mathrm{~mm}$ y 4,8 $\pm 1,8 \mathrm{~mm}$ en el lado derecho e izquierdo, respectivamente.

Tabla II. Hallazgos del foramen mental según sexo y edad en 215 radiografías panorámicas de pacientes chilenos. Se realizo la prueba ${ }^{2}$ de Pearson para detectar diferencias estadísticamente significativas.

\begin{tabular}{|c|c|c|c|c|c|c|c|c|c|c|c|}
\hline & & \multicolumn{5}{|c|}{ Visibilidad lado derecho } & \multicolumn{5}{|c|}{ Visibilidad lado izquierdo } \\
\hline & & \multicolumn{2}{|c|}{ Visible } & \multicolumn{2}{|c|}{ No visible } & \multirow{2}{*}{$\begin{array}{c}\text { Valor } \\
\mathbf{p}\end{array}$} & \multicolumn{2}{|c|}{ Visible } & \multicolumn{2}{|c|}{ No visible } & \multirow{2}{*}{$\begin{array}{c}\text { Valor } \\
\text { p }\end{array}$} \\
\hline & & $\%$ & $\mathbf{n}$ & $\%$ & $\mathbf{n}$ & & $\%$ & $\mathbf{n}$ & $\%$ & $\mathbf{n}$ & \\
\hline \multirow{3}{*}{ Sexo } & Mujer & 83,7 & 118 & 16,3 & 23 & \multirow{2}{*}{0,09} & 85,8 & 121 & 14,2 & 20 & \multirow{2}{*}{0,02} \\
\hline & Hombre & 91,9 & 68 & 8,1 & 6 & & 95,9 & 70 & 4,1 & 3 & \\
\hline & 21-35 años & 94,1 & 32 & 5,9 & 2 & \multirow{4}{*}{0,001} & 97,1 & 33 & 2,9 & 1 & \\
\hline \multirow{3}{*}{$\begin{array}{l}\text { Rango } \\
\text { etario }\end{array}$} & 36-50 años & 95,9 & 71 & 4,1 & 3 & & 94,6 & 70 & 5,4 & 4 & \multirow{3}{*}{0,01} \\
\hline & 51-65 años & 80 & 56 & 20 & 14 & & 85,5 & 59 & 14,5 & 10 & \\
\hline & > 65 años & 72,9 & 27 & 27 & 10 & & 78,4 & 29 & 21,6 & 8 & \\
\hline
\end{tabular}


Tabla III. Posición del foramen mental con relación a los dientes del lado derecho de la mandíbula. Se realizó la prueba x2 de Pearson para detectar diferencias estadísticamente significativas.

\begin{tabular}{|c|c|c|c|c|c|c|c|c|c|c|c|c|c|c|c|c|c|}
\hline & \multicolumn{7}{|c|}{ Posición supero-inferior } & \multicolumn{9}{|c|}{ Posición antero-posterior } \\
\hline & & \multicolumn{2}{|c|}{$\begin{array}{c}\text { Sobre } \\
\text { el ápice }\end{array}$} & \multicolumn{2}{|c|}{$\begin{array}{c}\text { En línea } \\
\text { con el }\end{array}$} & \multicolumn{2}{|c|}{$\begin{array}{c}\text { Bajo el } \\
\text { ápice }\end{array}$} & \multirow[t]{2}{*}{$\begin{array}{c}\text { Valor } \\
\mathbf{p}\end{array}$} & \multicolumn{2}{|c|}{$\begin{array}{l}\text { En línea } \\
\text { con 1er }\end{array}$} & \multicolumn{2}{|c|}{$\begin{array}{c}\text { Entre 1er } \\
\text { y } 2^{\text {do }} P M\end{array}$} & \multicolumn{2}{|c|}{$\begin{array}{l}\text { En línea } \\
\operatorname{con} 2^{d o}\end{array}$} & \multicolumn{2}{|c|}{$\begin{array}{l}\text { Posterior } \\
\text { al } 2^{\text {do }} P M\end{array}$} & \multirow[t]{2}{*}{$\begin{array}{c}\text { Valor } \\
\mathrm{p}\end{array}$} \\
\hline & & $\%$ & $\mathbf{n}$ & $\%$ & $\mathbf{n}$ & $\%$ & $\mathbf{n}$ & & $\%$ & $\mathbf{n}$ & $\%$ & $\mathbf{n}$ & $\%$ & n & $\%$ & $\mathbf{n}$ & \\
\hline \multirow[t]{2}{*}{ Sexo } & Mujer & 1,3 & 1 & 20 & 15 & 78,7 & 59 & 0,4 & 4 & 3 & 42,7 & 32 & 45,3 & 34 & 8 & 6 & 0,6 \\
\hline & Hombre & 3,9 & 2 & 25,5 & 13 & 70,6 & 36 & & 3,9 & 2 & 31,4 & 16 & 56,9 & 29 & 7,8 & 4 & \\
\hline \multirow{4}{*}{$\begin{array}{l}\text { Rango } \\
\text { etario }\end{array}$} & 21-35 años & 3,8 & 1 & 26,9 & 7 & 69,2 & 18 & 0,06 & 3,8 & 1 & 53,8 & 14 & 38,5 & 10 & 3,8 & 1 & 0,5 \\
\hline & 36-50 años & 3,8 & 2 & 23 & 12 & 73 & 38 & & 5,8 & 3 & 40,4 & 21 & 46,2 & 24 & 7,7 & 4 & \\
\hline & 51-65 años & 0 & 0 & 8,3 & 3 & 91,7 & 33 & & 2,8 & 1 & 27,8 & 10 & 58,3 & 21 & 11,1 & 4 & \\
\hline & $>65$ años & 1,3 & 1 & 20 & 15 & 78,7 & 59 & & 4 & 3 & 42,7 & 32 & 45,3 & 34 & 8 & 6 & \\
\hline
\end{tabular}

Tabla IV. Posición del foramen mental con relación a los dientes del lado izquierdo de la mandíbula. Se realizó la prueba x2de Pearson para detectar diferencias estadísticamente significativas.

\begin{tabular}{|c|c|c|c|c|c|c|c|c|c|c|c|c|c|c|c|c|c|}
\hline & \multicolumn{7}{|c|}{ Posición supero-inferior } & \multicolumn{9}{|c|}{ Posición antero-posterior } \\
\hline & & \multicolumn{2}{|c|}{$\begin{array}{l}\text { Sobre el } \\
\text { ápice }\end{array}$} & \multicolumn{2}{|c|}{$\begin{array}{l}\text { En línea } \\
\text { con el } \\
\text { ápice }\end{array}$} & \multicolumn{2}{|c|}{$\begin{array}{c}\text { Bajo el } \\
\text { ápice }\end{array}$} & \multirow[t]{2}{*}{$\begin{array}{c}\text { Valor } \\
\mathbf{p}\end{array}$} & \multicolumn{2}{|c|}{$\begin{array}{c}\text { En línea } \\
\text { con 1er } \\
\text { PM }\end{array}$} & \multicolumn{2}{|c|}{$\begin{array}{l}\text { Entre 1er } \\
\text { y } 2^{\text {do }} \text { PM }\end{array}$} & \multicolumn{2}{|c|}{$\begin{array}{c}\text { En línea } \\
\text { con } 2 \mathrm{do} \\
\text { PM }\end{array}$} & \multicolumn{2}{|c|}{$\begin{array}{c}\text { Posteri } \\
\text { or al 2do } \\
\text { PM }\end{array}$} & \multirow[t]{2}{*}{$\begin{array}{c}\text { Valor } \\
\mathbf{p}\end{array}$} \\
\hline & & $\%$ & $\mathbf{n}$ & $\%$ & n & $\%$ & $\mathbf{n}$ & & $\%$ & $\mathbf{n}$ & $\%$ & $\mathbf{n}$ & $\%$ & $\mathbf{n}$ & $\%$ & $\mathbf{n}$ & \\
\hline \multirow[t]{2}{*}{ Sexo } & Mujer & 0 & 0 & 36,2 & 25 & 63,8 & 44 & 0,2 & 7,2 & 5 & 50,7 & 35 & 36,2 & 25 & 5,8 & 4 & 0,2 \\
\hline & Hombre & 2 & 1 & 24 & 12 & 74 & 37 & & 12 & 6 & 36 & 18 & 50 & 25 & 2 & 1 & \\
\hline \multirow{4}{*}{$\begin{array}{l}\text { Rango } \\
\text { etario }\end{array}$} & 21-35 años & 0 & 0 & 40 & 10 & 60 & 15 & 0,3 & 0 & 0 & 56 & 14 & 44 & 11 & 0 & 0 & 0,5 \\
\hline & 36-50 años & 2,2 & 1 & 32,6 & 15 & 65,2 & 30 & & 13 & 6 & 37 & 17 & 43,5 & 20 & 6,5 & 3 & \\
\hline & 51-65 años & 0 & 0 & 18,9 & 7 & 81,1 & 30 & & 8,1 & 3 & 48,6 & 18 & 37,8 & 14 & 5,4 & 2 & \\
\hline & $>65$ años & 0 & 0 & 45,5 & 5 & 54,5 & 6 & & 18,2 & 2 & 36,4 & 4 & 45,5 & 5 & 0 & 0 & \\
\hline
\end{tabular}

\section{DISCUSIÓN}

La evidencia muestra variación en la detección y posición del FM según población estudiada. La detección del FM encontrada fue del 87,9\%, sin embargo, estudios previos en radiografías panorámicas han reportado porcentajes que fluctúan entre 77,8\% (Ngeow et al., 2010) y 97,6\% (Ngeow \& Yuzawati). Estudios en cráneos secos muestran la presencia bilateral del foramen mental en el $100 \%$ de los casos (Singh \& Srivastav; Oliveira et al., 2009; Ilayperuma et al., 2009). Se sugiere que la razón por la cual no es posible detectar el FM en radiografías panorámicas de algunos pacientes es la imposibilidad de distinguirlo del patrón trabecular circundante (Yosue \& Brooks). Además, se debe considerar la distorsión y amplificación inherentes a las radiografías panorámicas, la superposición de los dientes y los cambios del hueso mandibular propios de la extracción de dientes y aumento de edad.

En este estudio la detección del foramen en pacientes de entre 21 y 35 años fue de 94,1\% y 97,1\% (lado derecho e izquierdo, respectivamente), similar al 97,6\% reportado por Ngeow \& Yuzawati en una muestra cuyo promedio de edad fue de 24 años. Logramos visualizar el FM en un $72,9 \%$ y $78,4 \%$ (lado derecho e izquierdo, respectivamen- te) en pacientes de más de 65 años. Esta disminución en la visibilidad es aun más pronunciada en un estudio realizado por Ngeow et al. (2010), que sólo alcanzó al $45 \%$ en pacientes mayores de 50 años, mientras que Kuzmanovic et al. (2003) visualizaron los FM en el $88 \%$ de radiografías panorámicas de pacientes con un rango etario de 64 a 93 años. Esta disminución significativa de la visibilidad del FM a medida que aumenta la edad, puede deberse a que después de los 50 años existe un aumento en la porosidad del hueso cortical y una disminución de la masa ósea (Hildebolt, 1997), lo que causaría un aumento en la radiolucidez del hueso y la subsecuente falta de contraste con el FM, siendo esta última una estructura radiolúcida. Por otra parte, se encontró mayor hallazgo del FM en el sexo masculino (91,9\% y 95,9\% en el lado derecho e izquierdo, respectivamente), observando diferencias significativas solo en el lado izquierdo $(\mathrm{P}=0,02)$, lo que podría explicarse por la desmineralización y pérdida de masa ósea en individuos adultos, que es tres veces mayor en mujeres (Benson et al., 1991).

Otro aspecto importante fue que en este estudio la posición anteroposterior más frecuente se encontró en relación a la línea con el segundo premolar o entre los premolares 
Tabla V. Posición antero-posterior más frecuente del foramen mental, según diferentes muestras poblacionales.

\begin{tabular}{|c|c|c|c|}
\hline Población & Autor & Métodos & Posición \\
\hline Turquía & Yesilyurt et al. (2008) & Mandíbulas secas & En línea con el segundo premolar \\
\hline Brasil & Oliveira et al. (2009) & Mandíbulas secas & $\begin{array}{l}\text { En línea con el segundo premolar } \\
\text { Entre el 1er y } 2 \text { do premolar }\end{array}$ \\
\hline Brasil & Amorim et al. (2008) & Mandíbulas secas & En línea con el segundo premolar \\
\hline Sri Lanka & Ilayperuma et al. (2009) & Mandíbulas secas & En línea con el segundo premolar \\
\hline Jordania & Al-Khateeb et al. (2007) & Radiografías Panorámicas & Entre el 1er y 2 do premolar \\
\hline Arabia Saudita & al Jasser \& Nwoku (1998) & Radiografías Panorámicas & En línea con el segundo premolar \\
\hline India & Singh \& Srivastav (2010) & Mandíbulas secas & En línea con el segundo premolar \\
\hline Malasia & Ngeow \& Yuzawati (2003) & Radiografías Panorámicas & En línea con el segundo premolar \\
\hline Malasia & Igbigbi \& Lebona (2005) & Mandíbulas secas & En línea con el segundo premolar \\
\hline
\end{tabular}

del lado correspondiente. Estos resultados concuerdan con los resultados obtenidos en otras poblaciones (Tabla V). Si bien la mayoría de los datos se agrupan entre estas dos posiciones, existe un porcentaje (menor al 10\%) ubicado en línea con el primer premolar o posteriores al segundo premolar que no deben ser ignorados. No se observaron forámenes localizados anterior al eje longitudinal del primer premolar (Igbigbi \& Lebona; Yesilyurt et al.). Además, se observó una tendencia en relación a una posición más posterior del foramen a medida que aumenta la edad (Al-Khateeb et al.). La posición superoinferior más frecuente observada fue bajo los ápices de los dientes en relación al foramen, independiente del lado, sexo y edad del paciente (Tabla IV), coincidiendo con lo reportado por Al-Khateeb et al.

La distancia desde el centro del foramen mental y la línea mediana (D1) en este estudio fue de 35,3 mm en el lado derecho y $35,6 \mathrm{~mm}$ en el lado izquierdo, lo que es levemente superior a los $31,9 \mathrm{~mm}$ en el lado derecho y $32,9 \mathrm{~mm}$ en el lado izquierdo, encontrados por Fuentes $e t$ $a l$, en un estudio similar en pacientes chilenos adultos. Alves (2009) midió la distancia del margen anterior del foramen mental al pogonion en mandíbulas maceradas y el resultado fue de $25,58 \mathrm{~mm}$ en el lado derecho y 25,24 $\mathrm{mm}$ en el lado izquierdo. Si se considera la magnificación de las radiografías panorámicas, y que el punto de medición en este estudio fue desde el centro del foramen y no desde su margen anterior, se podría establecer que los resultados son similares. La distancia del centro del foramen al margen inferior de la mandíbula (D2) fue de 16,6 $\mathrm{mm}$ en el lado derecho y $17,1 \mathrm{~mm}$ en el lado izquierdo, cercanos a los 12,96 mm encontrados por Oliveira et al., si se considera una vez más la magnificación producida por las radiografías panorámicas.

La visualización del bucle mandibular en un 33,9\% de los casos estudiados fue menor que el 40,2\% reportado por Ngeow et al. (2009) y al 76\% obtenido por Arzouman et al. (1993), en radiografías panorámicas, pero es considerablemente superior al $11 \%$ encontrado por Jacobs et al. La identificación en cráneos secos ha sido reportada entre un $71 \%$ (Uchida et al.) y $100 \%$ (Arzouman et al.) y en tomografías computarizadas Cone-Beam, entre el $48 \%$ (Apostolakis \& Brawn, 2012) y el 84\% (Parnia et al., 2012). En este estudio, el hallazgo de esta referencia anatómica fue significativamente mayor en el lado izquierdo de la mandíbula ( $41 \%$ por sobre un $27 \%$ en el lado derecho) lo que coincide con los resultados de Arzouman et al. De igual manera, se determinó una longitud del bucle de 5,3 $\mathrm{mm}$ y de 4,8 $\mathrm{mm}$ en el lado derecho e izquierdo, respectivamente. Otros estudios en radiografías panorámicas han reportado longitudes desde 0,5 $\mathrm{mm}$ (Kuzmanovic et al.) hasta $5,7 \mathrm{~mm}$ (Apostolakis \& Brown). En algunas mandíbulas se han establecido longitudes de $6,95 \mathrm{~mm}$ (Arzouman et al.), a pesar que ésta es una medición realizada en toda la extensión del bucle y no sólo en su longitud antero-posterior.

Para evitar el daño neurovascular en la zona del foramen mental, principalmente por la eventual presencia del bucle del canal mandibular, algunos autores han propuesto un margen de seguridad de entre $4 \mathrm{~mm}$ (Kuzmanovic et al.) y $6 \mathrm{~mm}$ (Apostolakis \& Brawn) desde el borde medial del FM en dirección anterior. Los resultados de este estudio concuerdan con los valores propuestos por Apostolakis \& Brawn.

Las radiografías panorámicas convencionales tienen una alta capacidad para determinar presencia y posición del foramen mental, sobre todo en pacientes hombres de entre 21 y 50 años. El bucle del canal mandibular, sin embargo presenta una visualización en radiografías panorámicas considerablemente inferior a lo reportado en estudios en cráneos humanos (Neiva, Uchida, Arzouman). En los casos en que no sea posible identificar el FM o el bucle del canal mandibular se recomienda el uso de tomografías computarizadas (Garay \& Cantín, 2013; Martínez et al., 2013).

La anatomía de la zona circundante al foramen mental debe ser cuidadosamente analizada previo a cualquier tratamiento quirúrgico, debido a la gran variación en la posición del FM, según edad, sexo y lado. 
FUENTES, R.; CANTIN. M.; NAVARRO, P.; BORIE, E.; BELTRAN, V. \& BUCCHI, C. Characterization of anatomical structures using panoramic radiographs: the mental foramen. Int. J. Morphol., 32(4):1423-1429, 2014.

SUMMARY: Mental foramen (MF) is an important anatomical reference during surgery and implants. In order to meet the detectability and MF position, and describe it with respect to its anatomical relations, we analyzed 215 conventional panoramic radiographs. Furthermore, the presence and extent of mandibular canal loop was determined. The MF was detected in $87.9 \%$ of cases, with a greater percentage in men and a a younger age. The most frequent anteroposterior position of the MF was in relation to the longitudinal axis of the second premolar on the right, and between the longitudinal axes of the first and second premolar on the left. The superior-inferior position observed most frequently in both sexes and in all age groups was under the apex of the teeth. The average distance from the center of the MF to the midline was $35.3 \mathrm{~mm}$ and $35.6 \mathrm{~mm}$ in the right and left side, respectively. The average distance from the center of the mandibular MF margin was $16.6 \mathrm{~mm}$ on the right and $17.1 \mathrm{~mm}$ on the left. In relation to the mandibular canal loop, it was detected in $33.9 \%$ of cases and its extent was $5.3 \mathrm{~mm}$ and $4.8 \mathrm{~mm}$ on the right and left side, respectively. It is important to understand the anatomy, location of structures and to evaluate this area prior to any surgical procedure or dental implant, in order to avoid injuries to the MF, the mental nerve, anterior or posterior mandibular canal loop and inferior alveolar nerve.

radiography.

KEY WORDS: Mental foramen; Mandibular canal; Anterior mandibular canal loop; Inferior alveolar nerve; Panoramic

\section{REFERENCIAS BIBLIOGRÁFICAS}

al Jasser, N. M. \& Nwoku, A. L. Radiographic study of the mental foramen in a selected Saudi population. Dentomaxillofac. Radiol., 27(6):341-3, 1998.

Al-Khateeb T.; Al-Hadi Hamasha, A. \& Ababneh, K. T. Position of the mental foramen in a northern regional Jordanian population. Surg. Radiol. Anat., 29(3):231-7, 2007.

Alves, N. Study of the localization of the mental foramen in macerated mandibles with different degree of edentulism. Int. J. Odontostomat., 3(1):41-5, 2009.

Aminoshariae, A.; Su, A. \& Kulild, J. C. Determination of the location of the mental foramen: a critical review. J. Endod., 40(4):471-5, 2014.

Amorim, M. M.; Prado, F. P.; Borini, C. B.; Bittar, T. O.; Volpato, M. C.; Groppo, F. C. \& Caria, P. H. F. The mental foramen position in dentate and edentulous Brazilian's mandible. Int. J. Morphol., 26(4):981-7, 2008.

Arzouman, M. J.; Otis, L.; Kipnis, V. \& Levine, D. Observations of the anterior loop of the inferior alveolar canal. Int. J. Oral Maxillofac. Implants, 8(3):295-300, 1993.

Apostolakis, D. \& Brown, J. E. The anterior loop of the inferior alveolar nerve: prevalence, measurement of its length and a recommendation for interforaminal implant installation based on cone beam CT imaging. Clin. Oral Implants Res., 23(9):1022-30, 2012.

Benson, B. W.; Prihoda, T. J. \& Glass, B. J. Variations in adult cortical bone mass as measured by a panoramic mandibular index. Oral Surg. Oral Med. Oral Pathol., 71(3):349-56, 1991.
Fuentes, R.; Prieto, R.; Silva, H.; Bornhardt, T. \& Cuevas, F. Mandibular Morphometry in Adults by Panoramic Radiographic. Int. J. Morphol., 26(2):415-8, 2008.

Garay, I. \& Cantín, M. Accessory Mental Foramina Assessed by Cone-Beam Computed Tomography: Report of Unilateral and Bilateral Detection. Int. J. Morphol., 31(3):1104-8, 2013.

Hildebolt, C. F. Osteoporosis and oral bone loss. Dentomaxillofac. Radiol., 26(1):3-15, 1997.

Igbigbi, P. S. \& Lebona, S. The position and dimensions of the mental foramen in adult Malawian mandibles. West Afr. J. Med., 24(3):184-9, 2005.

Ilayperuma, I.; Nanayakkara, G. \& Palahepitiya, N. Morphometric analysis of the mental foramen in adult Sri Lankan mandibles. Int. J. Morphol., 27(4):1019-24, 2009.

Jacobs, R.; Mraiwa, N.; Van Steenberghe, D.; Sanderink, G. \& Quirynen, M. Appearance of the mandibular incisive canal on panoramic radiographs. Surg. Radiol. Anat., 26(4):329-33, 2004.

Kuzmanovic, D. V.; Payne, A. G.; Kieser, J. A. \& Dias, G. J. Anterior loop of the mental nerve: a morphological and radiographic study. Clin. Oral Implants Res., 14(4):464-71, 2003.

Martínez, F.; Olate, S.; Almeida, A. \& Vásquez, B. Unilateral Double Accessory Mental Foramen. Case Report. Int. J. Morphol., 31(4):1470-2, 2013.

Neiva, R. F.; Gapski, R. \& Wang, H. L. Morphometric analysis of implant-related anatomy in Caucasian skulls. J. Periodontol., 75(8):1061-7, 2004. 
FUENTES, R.; CANTIN. M.; NAVARRO, P.; BORIE, E.; BELTRAN, V. \& BUCCHI, C. Caracterización de estructuras anatómicas mediante radiografías panorámicas: El foramen mental. Int. J. Morphol., 32(4):1423-1429, 2014.

Ngeow, W. C. \& Yuzawati, Y. The location of the mental foramen in a selected Malay population. J. Oral Sci., 45(3):171-5, 2003.

Ngeow, W. C.; Dionysius, D. D.; Ishak, H. \& Nambiar, P. A radiographic study on the visualization of the anterior loop in dentate subjects of different age groups. J. Oral Sci., 51(2):231-7, 2009.

Ngeow, W. C.; Dionysius, D. D.; Ishak, H. \& Nambiar, P. Effect of ageing towards location and visibility of mental foramen on panoramic radiographs. Singapore Dent. J., 31(1):15-9, 2010.

Oliveira, Jr. E. M.; Araújo, A. L. D.; Da Silva, C. M. F.; SousaRodrigues, C. F. \& Lima, F. J. C. Morphological and morphometric study of the mental foramen on the M-CP-18 jiachenjiang point. Int. J. Morphol., 27(1):231-8, 2009.

Olivier, E. The inferior dental canal and its nerve in the adult. $\mathrm{Br}$. Dent. J., 49:356-8, 1928.

Parnia, F.; Moslehifard, E.; Hafezeqoran, A.; Mahboub, F. \& Mojaver-Kahnamoui, H. Characteristics of anatomical landmarks in the mandibular interforaminal region: a conebeam computed tomography study. Med. Oral Patol. Oral Cir. Bucal., 17(3):e420-5, 2012.

Standring, S.; Ellis, H.; Healy, J. C.; Johnson, D.; Williams, A.; Collins, P. \& Wigley, C . Gray's Anatomy. The Anatomical Basis of Clinical Practice. $39^{\text {th }}$ ed. London, Elsevier Churchill Livingstone, 2005.

Singh, R. \& Srivastav, A. Study of position, shape, size and incidence of mental foramen and accessory mental foramen in Indian adult human skulls. Int. J. Morphol., 28(4):1141-6, 2010.

Uchida, Y.; Noguchi, N.; Goto, M.; Yamashita, Y.; Hanihara, T.; Takamori, H.; Sato, I.; Kawai, T. \& Yosue, T. Measurement of anterior loop length for the mandibular canal and diameter of the mandibular incisive canal to avoid nerve damage when installing endosseous implants in the interforaminal region: a second attempt introducing cone beam computed tomography. J. Oral Maxillofac. Surg., 67(4):744-50, 2009.

Yesilyurt, H.; Aydinlioglu, A.; Kavakli, A.; Ekinci, N.: Eroglu, C.; Hacialiogullari, M. \& Diyarbakirli, S. Local differences in the position of the mental foramen. Folia Morphol. (Warsz.), 67(1):32-5, 2008 .

Yosue, T. \& Brooks, S. L. The appearance of mental foramina on panoramic radiographs. I. Evaluation of patients. Oral Surg. Oral Med. Oral Pathol., 68(3):360-4, 1989.

\author{
Dirección para Corresponcia: \\ Dr. Ramón Fuentes Fernández \\ Facultad de Odontología \\ Universidad de La Frontera \\ Manuel Montt 112 \\ Casilla 54-D, Temuco \\ CHILE
}

Email: ramon.fuentes@ufrontera.cl

Recibido : 03-06-2014

Aceptado: 12-09-2014 